\title{
Identification of Japanese Lecanorchis (Orchidaceae) Species in Fruiting Stage
}

\author{
Hirokazu Fukunaga ${ }^{1}$, Yutaka Sawa ${ }^{2} \&$ Shinichiro Sawa ${ }^{3}$ \\ ${ }^{1}$ Tokushima-cho, Tokushima city, Tokushima, Japan \\ ${ }^{2}$ Sawa Orchid Laboratory, Ikku, Kochi city, Kochi, Japan \\ ${ }^{3}$ Kumamoto University, Graduate school of Science and Technology, Kumamoto, Japan \\ Correspondence: Shinichiro Sawa, Kumamoto University, Graduate school of Science and Technology, \\ Department of Sciences, 2-39-1 Kurokami, Kumamoto 860-8555, Japan. Tel: 81-96-342-3439. E-mail: \\ sawa@sci.kumamoto-u.ac.jp
}

Received: December 4, 2013 Accepted: January 2, 2014 Online Published: January 7, 2014

doi:10.5539/ijb.v6n2p1 URL: http://dx.doi.org/10.5539/ijb.v6n2p1

\begin{abstract}
Plants of Lecanorchis species are heteromycotrophic and they lack green leaves. Although flowering time is short, plants with fruits can be easily found in the forests. Here we discuss the features of nine taxa namely $L$. triloba, L. trachycaula, L. nigricans, L. amethystea, L. kiusiana, L. suginoana, L. japonica, L. hokurikuensis, and L. flavicans var. acutiloba. From the detailed phenotypes of aerial parts of fruiting plants, we propose a method to identify the Japanese Lecanorchis species.
\end{abstract}

Keywords: Lecanorchis, Japanese orchids, Orchidaceae, diagnosis method, fruiting plants

\section{Introduction}

Lecanorchis Blume (Orchidaceae) comprises a group of mycoparasitic plants with numerous clustered, tuberous roots and an erect, branched or unbranched stem (Blume, 1856). The genus comprises about thirty taxadistributed across a large area between Southeast Asia, Taiwan, New Guinea, and Japan (Garay \& Sweet, 1974; Seidenfaden, 1978; Lin, 1987; Hashimoto, 1990; Pearce \& Cribb, 1999; Szlachetko \& Mytnik, 2000; Averyanov, 2005; Sing-chi, Cribb, \& Gale, 2009; Suddee \& Pedersen, 2011; Tsukaya \& Okada, 2013). In Japan, ten species and/or varieties are reported (Honda, 1931; Tuyama, 1955; Masamune, 1963; Ohwi, 1965; Satomi, 1982; Tuyama, 1982; Hashimoto, 1990; Serizawa, 2005; S. Sawa, Fukunaga, \& Y. Sawa, 2006; Fukunaga, S. Sawa, \& Y. Sawa, 2008; BG Plants, 2013). Most of Orchidaceaemembers that lack green leaves produce stem, flowers and seeds, and they wither and die in a short time. However, Lecanorchis species maintain withered plants at upper ground for a longer period, and the fruiting plants can be easily found in the forests. So, a diagnostic method using fruitingspecimens is needed to identify the species of Lecanorchis.

Here we have provided a simple method to identify eight Lecanorchis species namely L. triloba, L. trachycaula, L. nigricans, L. amethystea, L. kiusiana, L. suginoana, L. japonica, and L. hokurikuensis, and one variety, L. flavicans var. acutiloba. Other Japanese Lecanorchis taxa namely L. flavicans Fukuy., L. virella T. Hashim., L. japonica var. tubufolmis T. Hashim., L.nigricans var. patipetala Y. Sawa and L. nigricans var. yakushimensis T. Hashim. are uncommon and excluded from this diagnosis.

\section{Materials and Methods}

Field surveys were conducted in Kochi, Tokushima, Ehime, Kagawa, Osaka, Wakayama, Hyogo, Kyoto, Shimane, Shizuoka, Aichi, Gifu, Fukui, Ishikawa, Toyama, Fukuoka, Kagoshima, Miyazaki, and Okinawa prefectures. Herbarium specimens were deposited at MBK, OSA, KANA, TUAT, and mainly at TI herbaria.

Capsule angle was measured between stem and peduncle. Specimens of opened capsules were reconstituted by treating with hot water for a few second.

Obtuse excrescence produced on capsulesand upper part of infructescence is sometimes used as a taxonomic character (Masamune, 1963; Satomi, 1982) (Figure 1A). However, in some cases the phenotype was not observed. Further, the phenotype is induced by sapping by Flatidae (Figure 1B), aphid (Figure 1C), or scale insect (Sawa, 1980), and in some cases, this obtuse excrescence was not considered as a taxonomic character 
(Serizawa, 2005). In this article, we exclude this phenotype as a character for classification.
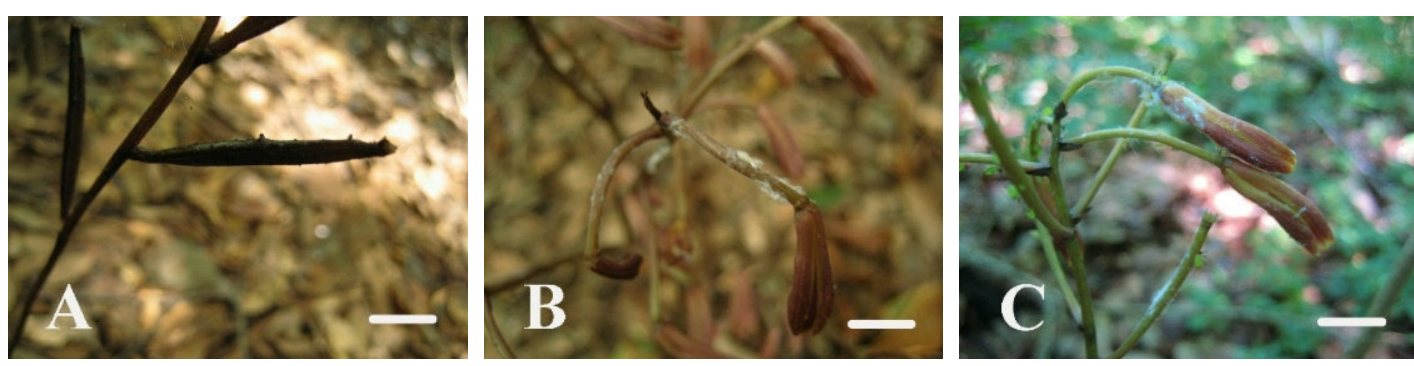

Figure 1. Obtuse excrescence and insects. A: Obtuse excrescence produced in L. hokurikuensis. B: Flatidae on the peduncle of L. hokurikuensis. C: Aphid on the peduncle of L. hokurikuensis. Scale bar: $1 \mathrm{~cm}$

\section{Results and Discussion}

Stem length, stem width, length of infructescence (stem with fruits), peduncle length, bract length, calyculus length, capsule number, color of capsule and stem, protuberance on capsule and stem, capsule angle, capsule shape, and branching pattern were examined by using eight Lecanorchis species, L. triloba, L. nigricans, $L$. amethystea, L. kiusiana, L. suginoana, L. japonica, and L. hokurikuensis, and one variety, L. flavicans var. Acutiloba (Table 1).

Table 1. Japanese Lechanorchis characters

\begin{tabular}{|c|c|c|c|c|c|c|c|c|c|}
\hline & L. triloba & L.trachycaula & L.nigricans & L.amethystea & $\begin{array}{l}\text { L.flavicans var. } \\
\text { acutiloba }\end{array}$ & L.kiusiana & L.suginoana & L. japonica & L.hokurikuensis \\
\hline \multirow[t]{2}{*}{$\begin{array}{l}\text { stem length } \\
\text { (average) } \\
(\mathrm{cm})\end{array}$} & $\begin{array}{c}14.5-42.0 \\
(34.6)\end{array}$ & $\begin{array}{c}23.5-41.7 \\
(32.1)\end{array}$ & $\begin{array}{c}11.4-27.7 \\
(19.1)\end{array}$ & $\begin{array}{c}22.2-42.7 \\
(33.6)\end{array}$ & $\begin{array}{c}14.0-35.1 \\
(23.3)\end{array}$ & $\begin{array}{c}3.9-25.4 \\
(17.3)\end{array}$ & $\begin{array}{c}12.0-29.8 \\
(20.5)\end{array}$ & $\begin{array}{c}21.2-45.2 \\
(30.9)\end{array}$ & $\begin{array}{c}17.6-50.4 \\
(30.5)\end{array}$ \\
\hline & $\mathrm{n}=8$ & $\mathrm{n}=8$ & $\mathrm{n}=38$ & $\mathrm{n}=12$ & $n=13$ & $\mathrm{n}=246$ & $\mathrm{n}=38$ & $\mathrm{n}=18$ & $\mathrm{n}=59$ \\
\hline \multirow{2}{*}{$\begin{array}{l}\text { stem diameter } \\
(\mathrm{mm})\end{array}$} & $0.8-2.2$ & $0.5-3.5$ & $1.0-2.2$ & $1.4-2.5$ & $0.5-1.3$ & $0.5-2.2$ & $1.7-3.5$ & $1.0-2.6$ & $1.3-3.6$ \\
\hline & $\mathrm{n}=8$ & $\mathrm{n}=8$ & $\mathrm{n}=38$ & $\mathrm{n}=12$ & $\mathrm{n}=13$ & $\mathrm{n}=246$ & $\mathrm{n}=38$ & $\mathrm{n}=18$ & $\mathrm{n}=59$ \\
\hline \multirow[t]{2}{*}{$\begin{array}{l}\text { inflorescence } \\
\text { length (average) } \\
\text { (cm) }\end{array}$} & $\begin{array}{c}1.9-5.8 \\
(4.0)\end{array}$ & $\begin{array}{l}2.8-15.0 \\
(6.1)\end{array}$ & $\begin{array}{l}1.7-16 \\
(5.2)\end{array}$ & $\begin{array}{c}8.5-17.0 \\
(13.1)\end{array}$ & $\begin{array}{c}1.0-6.0 \\
(3.2)\end{array}$ & $\begin{array}{l}1.5-10 \\
(4.6)\end{array}$ & $\begin{array}{c}3.5-8.0 \\
(5.6)\end{array}$ & $\begin{array}{c}2.0-14.0 \\
(7.1)\end{array}$ & $\begin{array}{c}3.0-8.5 \\
(5.7)\end{array}$ \\
\hline & $\mathrm{n}=8$ & $\mathrm{n}=17$ & $\mathrm{n}=27$ & $\mathrm{n}=9$ & $\mathrm{n}=11$ & $\mathrm{n}=17$ & $\mathrm{n}=5$ & $\mathrm{n}=11$ & $\mathrm{n}=8$ \\
\hline \multirow[t]{2}{*}{$\begin{array}{l}\text { capsule length } \\
\text { (average) } \\
\text { (cm) }\end{array}$} & $1.3-1.7(1.4)$ & $2.4-3.2(2.7)$ & $\begin{array}{l}2.1-3.6 \\
(2.6)\end{array}$ & $\begin{array}{l}1.9-3.3 \\
(2.6)\end{array}$ & $\begin{array}{l}2.3-2.7 \\
(2.5)\end{array}$ & $\begin{array}{l}1.9-3.0 \\
(2.4)\end{array}$ & $\begin{array}{l}2.0-3.0 \\
(2.7)\end{array}$ & $\begin{array}{c}3.1-4.4 \\
(3.7)\end{array}$ & $\begin{array}{c}3.1-4.8 \\
(3.8)\end{array}$ \\
\hline & $\mathrm{n}=24$ & $\mathrm{n}=5$ & $\mathrm{n}=66$ & $\mathrm{n}=51$ & $\mathrm{n}=6$ & $\mathrm{n}=381$ & $\mathrm{n}=72$ & $\mathrm{n}=15$ & $\mathrm{n}=111$ \\
\hline \multirow[t]{2}{*}{$\begin{array}{l}\text { peduncle length } \\
(\mathrm{mm})\end{array}$} & $0.5-1.5$ & $0.5-2.5$ & $1.5-2.5$ & $1.5-2.0$ & $1-1.5$ & $0.5-5.5$ & $2.0-3.5$ & $1.0-7.0$ & $2.0-7.5$ \\
\hline & $\mathrm{n}=24$ & $\mathrm{n}=5$ & $\mathrm{n}=15$ & $\mathrm{n}=51$ & $\mathrm{n}=3$ & $\mathrm{n}=156$ & $n=72$ & $\mathrm{n}=15$ & $\mathrm{n}=81$ \\
\hline \multirow[t]{2}{*}{$\begin{array}{l}\text { bract length } \\
\qquad(\mathrm{mm})\end{array}$} & $1.5-2.2$ & $1.4-2.5$ & $1.3-3.0$ & $1.8-2.5$ & $1.2-2.2$ & $1.7-5.2$ & $2.2-4.2$ & $2.2-6.0$ & $3.0-5.5$ \\
\hline & $\mathrm{n}=6$ & $\mathrm{n}=6$ & $\mathrm{n}=6$ & $\mathrm{n}=6$ & $\mathrm{n}=6$ & $\mathrm{n}=6$ & $\mathrm{n}=6$ & $\mathrm{n}=6$ & $\mathrm{n}=6$ \\
\hline \multirow[t]{2}{*}{$\begin{array}{l}\text { calyculus } \\
\text { length }(\mathrm{mm})\end{array}$} & $0.4-1.3$ & $0.5-1.4$ & $0.5-1.5$ & $0.3-1.0$ & $0.3-1.0$ & $0.5-1.2$ & $0.5-1.0$ & $0.5-2.2$ & $0.7-1.5$ \\
\hline & $\mathrm{n}=6$ & $\mathrm{n}=6$ & $\mathrm{n}=6$ & $\mathrm{n}=6$ & $\mathrm{n}=6$ & $\mathrm{n}=6$ & $\mathrm{n}=6$ & $\mathrm{n}=6$ & $\mathrm{n}=6$ \\
\hline \multirow{3}{*}{$\begin{array}{l}\text { capsule number } \\
\text { (average) }\end{array}$} & $3-14$ & $1-11$ & $2-11$ & $3-15$ & $1-7$ & $1-7$ & $3-6$ & $4-10$ & $2-10$ \\
\hline & (6.7) & (5.3) & (5.7) & (6.4) & (3.9) & (3.6) & (4.7) & $(6.2)$ & (5) \\
\hline & $\mathrm{n}=11$ & $\mathrm{n}=10$ & $\mathrm{n}=42$ & $\mathrm{n}=11$ & $\mathrm{n}=13$ & $\mathrm{n}=248$ & $\mathrm{n}=36$ & $\mathrm{n}=18$ & $\mathrm{n}=60$ \\
\hline \multirow[t]{2}{*}{$\begin{array}{c}\text { capsule angle to } \\
\text { stem }\end{array}$} & $10-45^{\circ}$ & $20-70^{\circ}$ & $0-75^{\circ}$ & $5-60^{\circ}$ & $5-70^{\circ}$ & $0-20^{\circ}$ & $0-30^{\circ}$ & $0-35^{\circ}$ & $5-90^{\circ}$ \\
\hline & $\mathrm{n}=6$ & $\mathrm{n}=6$ & $\mathrm{n}=6$ & $\mathrm{n}=6$ & $\mathrm{n}=6$ & $\mathrm{n}=6$ & $\mathrm{n}=6$ & $n=6$ & $\mathrm{n}=6$ \\
\hline
\end{tabular}



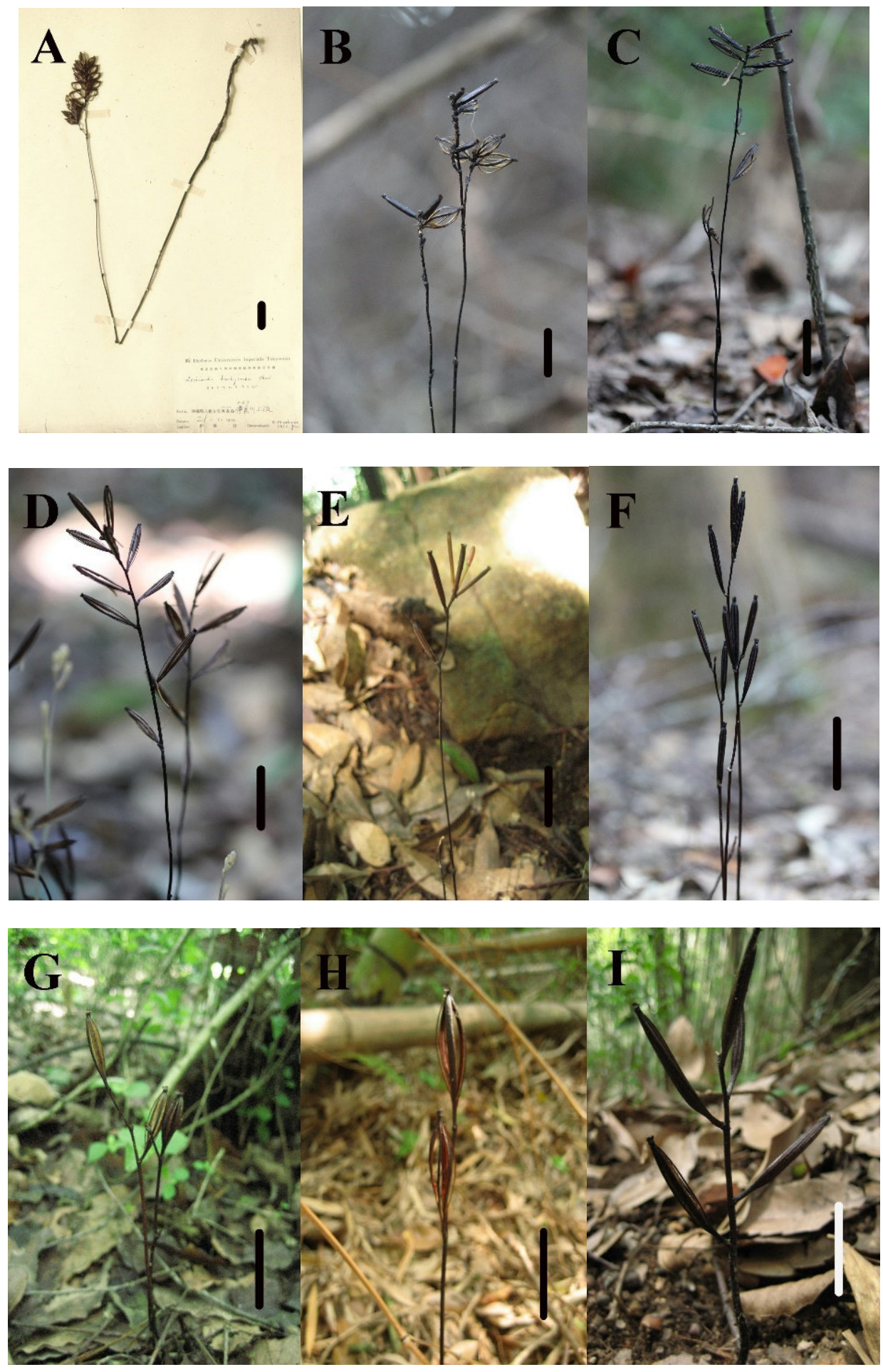

Figure 2. Fruiting plants of Lecanorchis spp. A: L. triloba, B: L. trachycaula, C: L.nigricans, D: L. amethystea, E: L. flavicans var. acutiloba. F: L. kiusiana, G: L. suginoana, H: L. japonica, I: L. hokurikuensis. Scale bar: $3 \mathrm{~cm}$ 

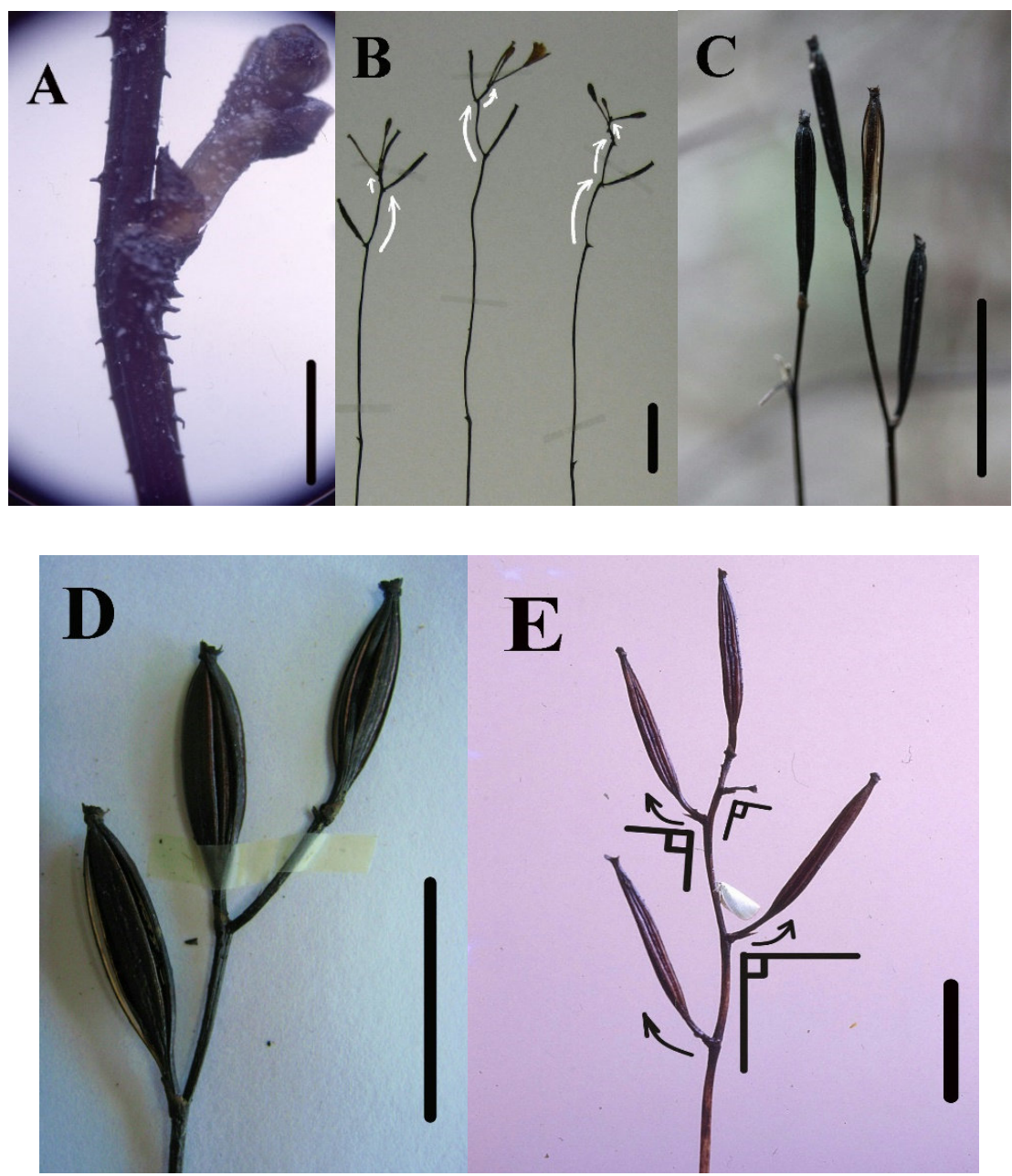

Figure 3. Characteristic phenotypes of Lecanorchis. A: Pointed, short prickle-like protuberances of $L$. trachycaula. B: Curved stem of L. flavicans var. acutiloba. C: Thin cylindrical to fusiform capsule of $L$. kiusiana. D: Roundish fusiform capsule of L. suginoana. E: Curved peduncle with a bract in $45-90^{\circ}$ of L. hokurikuensis. Scale bar: $5 \mathrm{~mm}$ (A) $3 \mathrm{~cm}$ (B-E)

\subsection{Lecanorchistriloba J. J. Sm.}

Lecanorchistriloba J. J. Sm. in Bull. Dep. Agric. Indes Neerl. 19: 26. 1908; Hashimoto in Ann. Tsukuba Bot. Gard. (9): 35. 1990; T. C. Hsu \& S. W. Chung in Taiwania 54: 83. 2009; L. brachycalpa Ohwi, in Acta Phytotax. Geobot. 7: 35. 1938; Tuyama in J. Jap. Bot. 30: 184. ff. 3 c\&d. 1955; Garay \& Sweet, Orchids of southern Ryukyu Islands 52. f.3-k. 1974; L. multiflora var. brachycalpa (Ohwi) T. Hashim., Hashimoto in Ann. Tsukuba Bot. Gard. (8): 6. 1989. (Figure 2A).

Characteristic features: Short infructescence, short peduncles, and small capsules.

Stems $14.5-42 \mathrm{~cm}$ long, 0.8-2.2 mm in diameter, solitary, rarely branched, black to brownish black. Scale leaves 3-4 mm long, acute triangle. Infructescence (stem with fruits) 1.9-5.8 cm long, with 3-14 fruits. Bract 1.5-2.2 $\mathrm{mm}$ long, acute, round to triangle. Capsule $1.3-1.7 \mathrm{~cm}$ long, fusiform, $10-45^{\circ}$ in capsule angle, densely produced at the top. Peduncle $0.5-1.5 \mathrm{~mm}$ long, Calyculus $0.4-1.3 \mathrm{~mm}$ long, irregularly denticulate.Fruits black to brownish black.

\section{Specimen Examined:}

Y. Taira 158, Jan. 22, 1938, Type of L. brachycalpa Ohwi (KYO); Y. Kimura s. n., Oct. 13, 1940 (TI); H. Ito s. n., Oct. 21, 1936 (TI); T. Hashimoto s. n., Feb. 19, 1990 (TNS-9504619); Y. Hanei s. n., May 29, 1989 (TNS-9504456); Y. Hanei s. n., Oct. 11, 1990 (TNS-9504683); T. Hashimoto s. n., Feb. 20, 1990 (TNS-9504620); T. Hashimoto, Feb. 20, 1990 (TNS-9504621); T. Amano 7735, Nov. 27, 1957 (TNS-139953); M. Tawada s. n., 
Dec. 28, 1941 (TNS-226051).

\subsection{Lecanorchis trachycaula Ohwi}

Lecanorchis trachycaula Ohwi, in Fl. Jap., ed. Rev. 1438. 1965; Hatus., Fl. Ryukyus 808. 1971; Satomi in Satake et al., Wild Fl. Jap. Herb. 1: 206. 1982; Hashimoto in Ann. Tsukuba Bot. Gard. 9: 19. 1990. (Figures 2B $\& 3 \mathrm{~A})$.

Characteristic features: Pointed, short prickle-like protuberances were found on stem and capsules, and the protuberances were obvious in later capsule stages (Figure 3A). Furthermore, non-glossy black capsules were produced laterally except for the uppermost and the lowermost capsules, and the stems were frequently branched.

Stems $23.5-41.7 \mathrm{~cm}$ long, $0.5-3.5 \mathrm{~mm}$ in diameter, 2-4 order branched, non-glossy black to purplish black, occasionally with pointed protuberances. Scale sheath $3-5 \mathrm{~mm}$ long. Infructescence $2.8-15 \mathrm{~cm}$ long, with 1-11 fruits. Bract 1.4-2.5 mm long, round to triangle. Capsule $2.4 \mathrm{~cm}-3.2 \mathrm{~cm}$ long, fusiform, $20-70^{\circ}$ in capsule angle except for the uppermost and the lowermost capsules, with occasional protuberances. Peduncle $0.5-2.5 \mathrm{~mm}$ long, Calyculus 0.5-1.4 mm long, irregularly denticulate.Fruits non-glossy black.

Specimen Examined:

C. Abe 49821, Jun. 11, 1974 (TKPM-214352); S. Takafuzi s. n., Oct. 27, 1963 (KANA); T. Hashimoto s. n., Feb. 24, 1990 (TNS-940462); J. Nakayama s. n., Aug. 10, 1936 (TUS); F. Maekawa s. n., Jun. 16, 1976 (TUAT-87954); S. Sugaya s. n., Aug. 14, 1958 (TUS-26508); S. Sugaya s. n., Aug. 15, 1958 (TUS-26509); N. Satomi s. n., Jul. 20, 1962 (KANA-158148).

\subsection{Lecanorchis nigricans Honda}

Lecanorchis nigricans Honda, in Bot. Mag. Tokyo 45: 470. 1931; Tuyama, in J. Jpn. Bot. 30: 184. ff. 3A\&B. 1955; Ohwi, Fl. Jap., Engl. Ed. 336. 1955; Kitamura et al., in Col. Ill. Herb. Pl. Jap. 3: 28. 1969; F. Maekawa, in Wild Orch. Jap. Col. 239. 1971; Satomi, in Satake et al., in Wild Fl. Jap. Herb. 1: 206.1982; Hashimoto, in Ann. Tsukuba Bot. Gard. (9): 27. 1990; Serizawa, Bunrui 5: 34. 2005. (Figure 2C).

Characteristicfeatures: Non-glossy black capsules were produced sideway except for top and bottom capsules. Protuberances were not produced compared with Lecanorchistrachycaula Ohwi.

Stems 11.4-27.7 cm long, 1.0-2.2 mm in diameter, non-glossy black, smooth surface, solitary, occasionally branched basally. Scale sheath $3 \mathrm{~mm}$ long. Infructescence $1.7-16 \mathrm{~cm}$ long, with 2-11 fruits. Bract 1.3-3 mm long, acute triangle. Capsule $2.1 \mathrm{~cm}-3.6 \mathrm{~cm}$ long, thin fusiform, smooth surfaced, $0-75^{\circ}$ in capsule angle except for the uppermost and the lowermost capsule. Peduncle 1.5-2.5 mm long, Calyculus 0.5-1.5 mm long, irregularly denticulate.Fruits non-glossy black.

Specimen Examined:

Type-K. Kashiyama s. n., 1931 (TI); T. Hashimoto s. n., Mar. 18, 1990 (TNS-9504625); E. Yamahata s. n., Jan. 6, 1976, det. by Y. Sawa (OSA), Y. Hanei s. n., Aug. 12, 1989 (TNS-9504539); Y. Kurosawa s. n., Aug. 8, 1978 (TUAT); K. Gunzi s. n., Oct. 13, 1963 (IBAR); T. Hino s. n., Oct. 16, 1928 det. by G. Murata (KYO), (OSA-19436), (KPM-NA-102387), (KPM-NA-162332), (KPM-NA-105066), (KPM-NA-73112), (KPM-NA-72853); Y. Sawa 2277, 16 Mar. 1999, (TI); I. Yamashita s. n., Oct. 16, 1965 (KANA-158148), T. Ogawa 26679 (KANA); Y. Sawa 2725, Aug. 18, 1998. (TI); Y. Sawa 2726, Aug. 18, 1998 (TI).

\subsection{Lecanorchis amethystea Y. Sawa, Fukunaga \& S. Sawa}

Lecanorchis amethystea Y. Sawa, Fukunaga \& S. Sawa, in Acta Phytotax. Geobot. 57: 123. 2006; Hsu, T.-C. \& S.-W. Chung, in Taiwania 55: 366. 2010. (Figure 2D).

Characteristicfeatures: Brown stems and capsules. Infructescences and stems were longer than that of Lecanorchisnigricans Honda (Sawa et al., 2006).

Stems $22.2-42.7 \mathrm{~cm}$ long, 1.4-2.5 $\mathrm{mm}$ in diameter, rarely branched basally, dark brown. Scale sheath $3-5 \mathrm{~mm}$ long, acute. Infructescence 8.5-17 cm long, with 3-15 fruits.Bract 1.8-2.5 mm long, acute triangle. Capsule 1.9 $\mathrm{cm}-3.3 \mathrm{~cm}$ long, fusiform, smooth surface, $5-60^{\circ}$ in capsule angle.Peduncle 1.5-2.0 mm long, Calyculus 0.3-1.0 $\mathrm{mm}$ long, irregular denticulate. Fruits dark brown.

Specimen Examined:

Type-Y. Sawa 1702, July 26, 1987 (TI); H. Fukunaga \& Y. Sawa 2, Aug. 29, 2000 (MBK); H. Fukunaga \& Y. Sawa 4, Aug. 29, 2000 (MBK); H. Fukunaga \& Y. Sawa 7, Aug. 29, 2000 (MBK); Y. Sawa 1864, Oct. 27, 1987 
(MBK); Y. Sawa 1869, Oct. 27, 1987 (MBK); Y. Sawa 1863, Oct. 27, 1987 (MBK).

\subsection{Lecanorchis flavicans Fukuy. var. acutiloba T. Hashim.}

Lecanorchis flavicans Fukuy. var. acutiloba T. Hashim., in Ann. Tsukuba Bot. Gard.(8): 8. 1989; Hashimoto, in Ann. Tsukuba Bot. Gard. (9): 25. 1990. (Figure 2E).

Characteristic features: Thin stems, short capsules and peduncles. Infructescence produced in zigzag with strong phototropism (Figure 3B).

Stems $14-35.1 \mathrm{~cm}$ long, $0.5-1.3 \mathrm{~mm}$ in diameter, solitary, rarely branched basally, growth in a zigzag way, black to brownish black. Infructescence 1-6 cm long, with 1-7 fruits. Bract 1.2-2.2 mm long. Capsule 2.3-2.7 cm long, thin fusiform, smooth surface, $5-70^{\circ}$ in capsule angle. Peduncle 1-1.5 mm long, Calyculus $0.3-1 \mathrm{~mm}$ long, irregular denticulate. Fruits brownish black.

Specimen Examined:

Y. Hanei s. n., July 26, 1991 (TNS-9507125); Y. Hanei s. n., July 26, 1991 (TNS-9507126); Y. Miyagi 5623, Jun. 25, 1966 (RYU-29181); Y. Miyagi 3394, Jun. 25, 1967 (RYU-18494); G. Murata et al. 865, det. by Y. Sawa (KYO), Y. Niiro s. n. (RYU-6422), Y. Sawa s. n. (TI).

\subsection{Lecanorchis kiusiana Tuyama}

Lecanorchis kiusiana Tuyama, in J. Jpn. Bot. 30: 182. 1955; Ohwi, in Fl. Jap., ed. Rev. 335. 1965; Chuma, in J. Jap. Bot. 55: 306. 1980; Satomi, in Satake et al., Wild Fl. Jap. Herb. 1: 205. 1982; Hashimoto, in Ann. Tsukuba Bot. Gard. (9): 13.1990; Serizawa, Bunrui 5: 37. 2005. (Figure 2F).

Characteristicfeatures: This is the smallest Lecanorchis species in Japan. Further, it produces narrowly tubular to fusiform capsules produced upward almost in parallel with the slender shoots (Figure 3C).

Stems 3.9-25.4 cm long, 0.5-2.2 $\mathrm{mm}$ in diameter, solitary, moderate gloss black to purplish black. Scale sheath 5-20 mm long, egg shaped at the base, acute at the top, smooth surface. Infructescence 1.5-10 cm long, with 1-7 fruits. Bract 1.7-5.2 mm long, triangle. Capsule 1.9-3.0 cm long, thin cylindrical to fusiform, smooth, parallel to stem, $0-20^{\circ}$ in capsule angle. Peduncle 0.5-5.5 mm long, Calyculus 0.5-1.2 mm long, irregular denticulate. Fruitsmoderate gloss black to purplish black.

Specimen Examined:

N. Satomi 1010, Oct. 4, 1951 det. by Y. Sawa (KANA-148801); T. Kobayashi 11259, July 27, 1988 det. by Y. Sawa (KYO); N. Satomi 22755, Mar. 29, 1964 det. by Y. Sawa (KANA148804); N. Satomi 16782, Mar. 28, 1961 det. by Y. Sawa (KANA148800); K. Inami s. n. , Jun. 17, 1975, det. by Y. Sawa \& G. Murata (KYO); H. Ono s.n., Nov. 17, 1968, det. by Y. Sawa (KAG); G. Ikeda s.n., Jul. 30, 1965, det. by Y. Sawa (KAG); I. Yamashita 3, Jun. 1979 (TUAT-87990), Y. Sawa 891 (OSA-47829, 167462); Y. Sawa \& H. Fukunaga 2572, Feb. 41999 (TI); Y. Sawa \& H. Fukunaga 2722, Mar. 16, 1999 (TI); Y. Sawa \& H. Fukunaga 2721, Mar. 161999 (TI); Y. Sawa \& H. Fukunaga 2729, Mar. 161999 (TI); Y. Sawa \& H. Fukunaga 2730, Mar. 161999 (TI); Y. Sawa \& H. Fukunaga 2732, Mar. 161999 (TI); Y. Sawa \& H. Fukunaga 2724, Mar. 161999 (TI); Y. Sawa \& H. Fukunaga 2731, Mar. 161999 (TI); Y. Sawa \& H. Fukunaga 2747, Mar. 171999 (TI); Y. Sawa \& H. Fukunaga 2748, Mar. 171999 (TI); Y. Sawa \& H. Fukunaga 2749, Mar. 171999 (TI); Y. Sawa \& H. Fukunaga 2279, Jan. 231999 (TI); Y. Sawa \& H. Fukunaga 2719, Aug. 61998 (TI); N. Satomi 22755 Mar. 29, 1964 (KANA-148804); N. Satomi 25033, Apr. 2, 1965 (KANA-147795); E. Ikeda 21674, Jun. 2, 1963 (KANA-134468); N. Satomi 16782, Mar. 28, 1961 (KANA-148800); N. Satomi 26845, Jul. 16, 1973 (KANA-148805); N. Satomi 148801, Oct. 4, 1951 (KANA-148801).

\subsection{Lecanorchis suginoana (Tuyama) Seriz}

Lecanorchis suginoana (Tuyama) Seriz, in Bunrui 5: 38. 2005; L. japonica var. sugioana Tuyama, in J. Jpn. Bot. 57: 211. 1982; L. kiusiana Tuyama var. suginoana Hashim, In Ann. Tsukuba Bot. Gard. (9): 18. 1990. (Figure 2G).

Characteristic features: Small plants with non-glossy brown to black, and round capsules (Figure 3D). Lower part of the shoot is thicker than that of L. kiusiana.

Stems $12-29.8 \mathrm{~cm}$ long, 1.7-3.5 mm in diameter, solitary, smooth surface, brown to black. Scale sheath 5-10 mm long. Infructescence 3.5-8 cm long, with 3-6 fruits.Bract 2.2-4.2 mm long, acute triangle.Capsule 2-3 cm long, roundish fusiform, $0-30^{\circ}$ in capsule angle. Peduncle 2-3.5 mm long. Calyculus $0.5-1 \mathrm{~mm}$ long, irregular denticulate, $0.5-1 \mathrm{~mm}$ long.Fruitsnon-glossy brownish black to black.

Specimen Examined: 
M. Wadas. n. Jul. 5, 1983 (TI), M. Tanaka 3856 (OSA), T. Umehara 4836 (OSA-91409); Y. Sawa 2524, Feb. 20, 1998 (TI); Y. Sawa 2524, Feb. 20, 1999 (TI); Y. Sawa 2524, Feb. 20, 2526, (TI); Y. Sawa 2527, Feb. 20, 1999, (TI); Y. Sawa 2533, Feb. 20, 1999 (TI); Y. Sawa 2763, Feb. 20, 1999 (TI); Y. Sawa 2766, Feb. 20, 1999 (TI); Y. Sawa 2538, Feb. 20, 1999 (TI); Y. Sawa 1439, May 18, 1987 (TI); Y. Sawa 1584, Jun. 12, 1987 (TI); Y. Sawa 872, May 23, 1987 (TI); Y. Sawa 875, May 18, 1987 (TI); Y. Sawa 871, May 18, 1987 (TI); Y. Sawa 945, Apr. 5, 1984 (TI); Y. Sawa 948, Apr. 5, 1984 (TI); Y. Sawa 949, Apr. 5, 1984 (TI); Y. Sawa 882, Jun. 15, 1984 (TI).

\subsection{Lecanorchis japonica Blume}

Lecanorchis japonica Blume, in Mus. Bot. Ludg. Bat. 2: 188. 1856; Kitamura et al., in Col. Ill. Herb. Pl. Jap. 3 : 28. 1969; F. Maekawa, in Wild Orch. Jap. Col. 236. 1971; Satomi in Satake et al., in Wild Fl. Jap. Herb. 1: 206. 1982; Hashimoto, in Ann. Tsukuba Bot. Gard. (9): 3. 1990; Serizawa Bunrui 5: 35. 2005. (Figure 2H).

Characteristic features: This is the largest Lecanorchis species in Japan. Further it produces capsules slant upward with straight peduncle and capsules.

Stems 21.2-45.2 cm long, 1-2.6 mm in diameter, solitary, black to brownish black. Scale Sheath 6-12 mm long. Infructescence 2-14 cm long, with 4-10 fruits. Bract 2.2-6 mm long, acute triangle. Capsule 3.1-4.4 cm long, fusiform, $0-35^{\circ}$ in capsule angle. Peduncle 1-7 mm long, straight, Calyculus $0.5-2.2 \mathrm{~mm}$ long, irregular denticulate. Fruits black to brownish black.

Specimen Examined:

K.Kawanabe 4705, Dec. 7, 1958 det. By Hatushima (KAG); Y. Sawa 1383, Dec. 26, 1986 (TUAT); S. Hatusima 20766 , May 14, 1957 (KAG); Y. Kashiyama s. n. Jan. 29, 1933 (KYO); K. Seto s. n., Jun. 28, 1962 (OSA); Y. Sawa \& H. Fukunaga 2718, Jan. 12, 1999 (TI); N. Satomi 25091, Apr. 25, 1965 (KANA-158145); N. Satomi 15232, Aug. 2, 1960 (KANA-158144); S. Kato s. n., Sep. 5, 1976 (KANA-145571); K. Aoki s. n., Jul. 1, 1970, (KANA-145570); N. Satomi 7269, Dec. 31, 1955 (KANA-148807); T. Omura s. n., (KANA-147707).

3.9 Lecanorchis hokurikuensis Masam.

Lecanorchis hokurikuensis Masam., in J. Geobot. 12: 69. 1963; Satomi in Satake et al., Wild Fl. Jap. Herb. 1: 205. 1982; Bunrui 5: 36. 2005; L. japonica Blume var. hokurikuensis (Masam.); Hashimoto, in Ann. Tsukuba Bot. Gard. (9): 7. 1990. (Figure 2I).

Characteristic features: The capsule angle $\left(45-90^{\circ}\right)$ to the shoot (Figure 3E), and peduncle is curved to upward (Figure 3E).

Stems 17.6-50.4 cm long, 1.3-3.6 mm in diameter, solitary, light brown to black. Scale Sheath 5-12 mm. Infructescence 3-8.5 cm long, with 2-10 fruits. Bract 3-5.5 mm long, acute triangle. $45-90^{\circ}$ in bract angle. Capsule 3.1-4.8 cm long, fusiform, 5-90 in capsule angle. Peduncle 2-7.5 mm long, upward. Calyculus 0.7-1.5 $\mathrm{mm}$ long, irregular denticulate. Fruits brown to brownish black.

Specimen Examined:

I. Yamashita s. n., Jun. 16, 1981, det. by Y. Sawa, (TUAT-85827); Suzuki, Nakamura, Moriguchi \& Tasaki s. n., Oct. 15, 1975, det. by Y. Sawa (IBAR); N. Satomi 27163, Nov. 19, 1972 (KANA-103715); A. Kimura s. n., Oct.3, 1931, det. by Y. Sawa (TUS-26500); F. Maekawa s. n., Jan. 1972, det. by Y. Sawa (TUAT-87864); F. Maekawa s.n., Mar. 4, 1972, det. by Y. Sawa (TUAT-87863); S. Tamaki s. n., Oct. 1970, det. by Y. Sawa (RYU-6832); Y. Miyagi 1763, Aug. 1972, det. by Y. Sawa (RYU-9966); N. Satomi s. n., Oct. 22, 1980 (KANA-94944); N. Satomi 27088, Oct. 22, 1978, (KANA-92137); N. Satomi 27028, Jul. 10, 1977 (KANA-146686); N. Satomi 27030, Jul. 10, 1977 (KANA-146687); S. Okuda s. n., May 31, 1970 (KANA-145579); G. Masamune 14169, Oct. 16, 1961 (KANA-44546); N. Satomi 15430, Sep. 8, 1960 (KANA-146680); N. Satomi 27163, Nov. 19, 1972 (KANA-103715); N. Satomi 26774, Nov. 3, 1972 (KANA-146685); N. Satomi 26941, Nov. 2, 1975 (KANA-146688); Y. Shirosaki 27024, (KANA-147792); N. Satomi s. n., Oct. 21, 1980 (KANA-97123); $N$. Satomi s.n., Sep. 20, 1953 (KANA-13921), N. Satomi 26942, Oct. 15, 1972 (KANA-146689, 81904), N. Satomi 27630, Jul. 22, 1986 (KANA-119847); Y. Sawa 2645, Jun. 12, 1998 (TI); Y. Sawa 2285, Jun. 12, 1998 (TI); Y. Sawa 2286, Jun. 12, 1998 (TI); Y. Sawa 2287, Jun. 12, 1998 (TI); N. Satomi 15181, Jul.1, 1960 (KANA-147790); N. Satomi 15661, Sep. 23, 1960 (KANA-146681); N. Satomi 20144, Jun. 25, 1962 (KANA-146682); N. Satomi 15167, Jul. 3, 1960 (KANA-146683); N. Kurosaki, 2009, Jul. 2, 1966 (KANA-668290); M. Ihara s. n., Jul. 1, 1957 (KANA-146684); Uzihara s. n., Sep. 4, 1955 (KANA-27649); N. Satomi 26846, Jul. 22, 1973 (KANA-145578); S. Sugaya s. n., Jun. 12, 1956 (KANA-145572); H. Kitami s. n., Jul. 5, 1968 (KANA-145575); Y. Ueno 12856, (KANA-145575). 
Key to the species of Japanese Lecanorchis (fruiting specimens)

1. Capsules 1.3-1.7 cm long .......... L. triloba

1. Capsules 1.9-4.8 cm long .......... 2

2. Stems branched at base .......... 3

2. Stems unbranched .......... 6

3. Capsule formation laterally .......... 4

3. Capsule formation upward ......... 5

4. Stems prickled .......... L. trachycaula

4. Stems smooth .......... L. nigricans

5. Infructescence straight, $8.5-17 \mathrm{~cm}$ long ......... L. amethystea

5. Infructescencecurved, 1-6 cmlong .......... L. flavicansvar. actiloba

6. Capsules 1.9-3.0 cm long ......... 7

6. Capsules 3.1-4.8 cm long .......... 8

7. Capsulesnarrowly cylindrical to fusiform .......... L. kiusiana

7. Capsules roundish fusiform .......... L. suginoana

8. Peduncles curved, with a bract in $45-90^{\circ} \ldots \ldots \ldots$. ... hokurikuensis

8. Peduncles straight, with a bract in $-45^{\circ} \ldots \ldots . .$. L. japonica

\section{References}

Averyanov, L. V. (2005). New Orchids from Vietnam. Rheedea, 15, 83-101.

BG Plants. (2013). In Web BG Plants, Y-list. Retrieved December 25, 2013, from http://bean.bio.chiba-u.jp/bgplants/ylist_main.html

Blume, C. L. (1856). Lecanorchis Bl. Mus. Bot. Ludg. Bat. 2, 188. Brill: Leiden.

Fukunaga, H., Sawa, S., \& Sawa, Y. (2008). A new form of Lecanorchiskiusiana. Orchid Rev., 116, 106-108.

Garay, L. A., \& Sweet, H. R. (1974). Orchids of southern Ryukyu Islands. Massachusetts, MA: Bot. Mus. Harvard Univ. Cambridge-Mass.

Hashimoto, T. (1990). A taxonomic review of the Japanese Lecanorchis (Orchidaceae). Ann. Tsukuba Bot. Gard., 9, 1-40.

Honda, M. (1931). Nuntia ad FloramJaponiae XIV. Bot. Mag. Tokyo, 45, 469-471, 493-494.

Lin, T. P. (1987). Native Orchids of Taiwan 3 (pp. 1-300). Taipei: Southern Materials Center.

Masamune, G. (1963). Lecanorchis hokurikuensis. J. Geobot., 12, 69.

Ohwi, J. (1965). Flora of Japan (Rev. ed.). Tokyo, Japan: Shibundo.

Pearce, N., \& Cribb, P. (1999). Notes Relating to the Flora of Bhutan: XXXVII. New species and records of Orchidaceae from Bhutan and India (Sikkim). Edinb. J. Bot., 56, 273-284. http://dx.doi.org/10.1017/S096042860000113X

Satomi, N. (1982). Ranka (Orchidaceae). In Y. Satake et al. (Eds.), Wild flowers of Japan: Herbaceous Plants I (pp. 187-235, pls. 170-208). Tokyo, Japan: Heibonsya Ltd., Pub.

Sawa Y. (1980). Reconsideration of obtuse excrescence as a taxonomic character. Annual meeting of the botanical society of Japan, 45, 54.

Sawa, S., Fukunaga, H., \& Sawa, Y. (2006). Lecanorchis amethystea (Orchidaceae), A new species from Kochi. Acta Phytotax. Geobot., 57, 123-128.

Seidenfaden, G. (1978). Orchid genera in Thailand VI. NeottioideaeLindl. Dansk Bot. Arkiv, 32(2), 1-195.

Serizawa, S. (2005). The genus Lecanorchis (Orchidaceae) in Aichi Prefecture, Central Honshu. Bunrui, 5, 33-38.

Sing-chi, C., Cribb, P. J., \& Gale, S. W. (2009). Lecanorchis. In Z. Y. Wu, P. H. Raven \& D. Y. Hong (Eds.), Flora of China (Orchidaceae) (Vol. 25, pp. 171-172). Beijing, Chinaand St. Louis, Missouri: Science Press 
and Missouri Botanical Garden Press.

Suddee, S., \& Pedersen, H. Æ. (2011). A new species of Lecanorchis (Orchidaceae) from Thailand. Taiwania, 56, 37-41.

Szlachetko, D. L., \& Mytnik, J. (2000). Lecanorchis seidenfadeni (Orchidaceae, Vanilloideae), a new orchid species from Malaya. Ann. Bot. Fennici, 37, 227-230.

Tsukaya, H., \& Okada, H. (2013). A new species of Lecanorchis (Orchidaceae, Vanilloideae) from Kalimantan, Borneo. Systematic Botany, 38, 69-74. http://www.bioone.org/doi/full/10.1600/036364413X662079

Tuyama, T. (1955). A new saprophytic orchid, Lecanorchis kiusiana. J. Jap. Bot., 30, 181-187.

Tuyama, T. (1982). Lecanorchis japonica Bl. var. suginoana Tuyama, a new variety, with comments on the other taxa of the genus. J. Jap. Bot., 57, 205-211.

\section{Copyrights}

Copyright for this article is retained by the author(s), with first publication rights granted to the journal.

This is an open-access article distributed under the terms and conditions of the Creative Commons Attribution license (http://creativecommons.org/licenses/by/3.0/). 